\title{
Constructing the Bridge from Isotropic to Anisotropic Pitches for Preparing Pitch-Based Carbon Fibers with Tunable Structures and Properties
}

Guanming Yuan,* Zheng Xue, Zhengwei Cui, Aidan Westwood, Zhijun Dong, Ye Cong, Jiang Zhang, Hui Zhu, and Xuanke Li*

Cite This: https://dx.doi.org/10.1021/acsomega.0c03226

ABSTRACT: Synthetic naphthalene pitches (SNPs) with isotropy and anisotropy were prepared by a simple thermal polycondensation method to fabricate pitch-based carbon fibers. The structural characteristic, thermal stability, phase-separation behavior, and melt-spinnability of the SNPs and the structural properties of the derived carbon fibers were systematically investigated. The results show that spinnable SNPs with controllable mesophase contents ranging from 0 to $100 \mathrm{vol} \%$ and softening points $\left(210-290^{\circ} \mathrm{C}\right)$ could be easily obtained by a nitrogenbubbling treatment to improve their thermal stability and meltspinnability by avoiding the phase separation of liquid crystal (LC) in the pitch. An experimental phase diagram of spinnability and mesophase


content is newly proposed for predicting the spinnability of a mesophase-containing pitch. The LC has a significant influence not only on the constituents, structure, and physical properties of the SNPs but also on the final structure and properties of the corresponding pitch-based carbon fibers. The low ash content (less than $0.15 \mathrm{wt} \%$ ) in the pitch precursor is found to have no obvious effect on the pitch spinnability and the mechanical properties of derivative large-diameter carbon fibers.

\section{INTRODUCTION}

Pitch-based carbon fibers are commonly divided into two classes: isotropic pitch and anisotropic mesophase pitch based. These two types of carbon fibers are different not only in carbonaceous precursors but also in their optical texture, microstructure, and physical properties. Mesophase pitch, with a typical nematic liquid crystalline structure and characteristic of easy graphitization, has long been regarded as an excellent precursor for making a wide variety of industrial and advanced engineering carbon products, especially for high-performance pitch-based carbon fibers. ${ }^{1-3}$ Recognized as an archetypal high-tech product with high added value, mesophase-pitchbased carbon fibers have been attracting extensive attention in both academia and industry. ${ }^{2,4,5}$ They are a promising and, at times, indispensable engineering material widely used in critical aerospace, defense, and military industries owing to their extremely high Young's modulus (up to the theoretical value of graphite, $1000 \mathrm{GPa}$ ), excellent axial electrical resistivity (as low as $1.0 \mu \Omega \mathrm{m}$ ), thermal conductivity (exceeding $1000 \mathrm{~W} /(\mathrm{m}$ $\mathrm{K})$ ), good high-temperature corrosion resistance, etc. ${ }^{2,6-8}$ This outstanding performance is a direct result of their highly crystalline graphitic structure and a high degree of continuous orientation parallel to the fiber axis, which is undoubtedly inherited from the well-ordered texture of liquid crystal (LC) in the pitch precursor. ${ }^{2,8}$ That is to say, the physical properties of pitch-based carbon fibers are mostly dominated by their ideal microstructure (i.e., preferred crystalline orientation), which is closely related to the unique characteristics (e.g., molecular structure, LC texture, physical properties, etc.) of the starting pitch. ${ }^{2,3}$ Therefore, the regulation and control of the final structure and properties of pitch-based carbon fibers could be expected to be achieved by modifying the nature of the pitch precursor under the premise of ensuring that other process conditions remain unchanged.

Nowadays, either thermal polycondensation of commercial coal-tar pitches and petroleum pitches (or even certain soluble fractions thereof) or catalytic polymerization of model aromatic compounds is commonly used to prepare a mesophase pitch. ${ }^{2,5,9-11}$ However, to date, it is still very difficult to produce cheap mesophase pitches on a large scale with high quality (especially for continuously melt-spinning high-performance carbon fibers). It is well known that the

Received: July 9, 2020

Accepted: August 6, 2020 
Table 1. Physical Properties of the NP Precursor and its TS Fraction

\begin{tabular}{|c|c|c|c|c|c|c|c|}
\hline \multirow[b]{2}{*}{ sample } & \multicolumn{3}{|c|}{ main fraction/wt $\%$} & \multirow[b]{2}{*}{$\mathrm{SP} /{ }^{\circ} \mathrm{C}$} & \multirow[b]{2}{*}{$\mathrm{CV} / \mathrm{wt} \%^{a}$} & \multirow[b]{2}{*}{$\mathrm{AC} / \mathrm{ppm}^{b}$} & \multirow[b]{2}{*}{$\mathrm{H} / \mathrm{C}(\mathrm{mol})$} \\
\hline & $\mathrm{CHS}$ & CHI-TS & TI & & & & \\
\hline NP & 22.4 & 63.1 & 14.5 & 124 & 32.5 & 790 & 0.76 \\
\hline NP-TS & 28.2 & 71.8 & & 103 & 28.6 & 170 & 0.79 \\
\hline
\end{tabular}

${ }^{a} \mathrm{CV}$ was determined by a $900{ }^{\circ} \mathrm{C}$ carbonization treatment for $1 \mathrm{~h} .{ }^{b} \mathrm{AC}$ was determined by an $850{ }^{\circ} \mathrm{C}$ burning treatment for $3 \mathrm{~h}$.

nature of carbonaceous precursors for the preparation of spinnable mesophase pitches is very crucial. Although commercial coal-tar pitches or petroleum pitches are cheap and easy to obtain, these pitch feedstocks are normally a complex mixture including some heteroatoms and inorganic ash $(\sim 0.2 \mathrm{wt} \%)$, from which it is difficult to prepare a spinnable mesophase pitch. $^{12-14}$ Over the last thirty years, small organic molecules (such as naphthalene) have been widely used to synthesize spinnable mesophase pitches by catalyzing polymerization with the volatile superacid, $\mathrm{HF}-\mathrm{BF}_{3}$. The resulting naphthalene-derived mesophase pitch possesses characteristics of high purity, controllable molecular structure, and more ideal physical properties. ${ }^{2,15}$ However, the severe corrosion problem and potential operating risk when using $\mathrm{HF}_{-} \mathrm{BF}_{3}$ as a catalyst unfortunately limit its widespread use (and such a mesophase pitch product is now no longer available from, e.g., Mitsubishi Gas Chemical Company). In the meantime, a milder catalyst, $\mathrm{AlCl}_{3}$, has been selectively used to achieve the anticipated catalytic polymerization effect despite residual traces of this catalyst inevitably remaining, intermingled in the mesophase pitch. ${ }^{2,16,17}$

It has been demonstrated that the formation, development, and transformation of liquid crystalline, optically anisotropic spheres (i.e., mesophase nucleation, growth, coalescence, and deformation and orientation) in an isotropic pitch matrix occur unconcertedly and inhomogeneously during the process of liquid-phase carbonization. ${ }^{18,19}$ Furthermore, it is not easy to obtain a 100 vol \% anisotropic mesophase pitch (i.e., bulk mesophase) both with a fine flow optical texture and an acceptable softening point (SP) less than $300{ }^{\circ} \mathrm{C}$ for subsequent fiber spinning. This mainly depends on the carbonaceous precursors used and the adoption of suitable thermal reaction conditions. ${ }^{18-20}$ Hence, many studies have focused on the preparation and application of a partial LC pitch. $^{21-24}$ However, the two phases (i.e., isotropic and anisotropic components) in a partial mesophase pitch or an isotropic pitch containing some mesophase are commonly not compatible (or coexistent) and become extremely unstable in a molten state, resulting in obvious mesophase phase-separation behavior, particularly in the case of a partial mesophase pitch made from multiple heterogeneous precursors under conditions of gravity sedimentation or centrifugation (contrarily as an effective strategy to concentrate the mesophase). ${ }^{23,25-27}$ According to a common viewpoint, a spinnable mesophase pitch should meet the essential requirements of a high LC content (nearly 100 vol \%), a suitable SP of $\sim 260{ }^{\circ} \mathrm{C}$, and a low ash content $(\mathrm{AC})$ of less than $\sim 100 \mathrm{ppm}$. Although there have been a few studies on blending two types of pitches for carbon fiber preparation, ${ }^{21,27-30}$ the influence of mesophase contents and the amount of $\mathrm{AC}$ in various spinnable pitches derived from a single pitch raw material on pitch spinnability and derived fiber properties is still not clearly known. It is clear that, so far, there has been a lack of research on a "bridge" connecting an isotropic pitch and a bulk mesophase pitch (when used as spinnable pitches starting from the same pitch precursor) so as to provide a systematic and comprehensive study on pitch-based carbon fibers.

In this work, various synthetic naphthalene pitches (SNPs) with different LC contents and SPs were prepared by controlling air oxidation and direct thermal polycondensation treatments of a lab-made naphthalene pitch (NP) in an autoclave reactor. Melt-spinnable synthetic pitches with representative $\mathrm{LC}$ contents $(0,5,50,75,98$, and 100 vol \%) and controllable SPs ranging from 210 to $290{ }^{\circ} \mathrm{C}$ could be adjusted by further nitrogen-bubbling treatment on thermally polymerized pitches to volatilize some light fractions to achieve the phase-separation control of LC in the SNPs. The influence of LC contents (including the optical orientation texture) not only on the constituents, structure, and properties of the spinnable pitches but also on the morphology, microstructure, and physical properties of the derived carbon fibers was comprehensively researched. Large-diameter $(\Phi \sim 20 \mu \mathrm{m})$ carbon fibers prepared from various spinnable SNPs were selected as model products (owing to their relatively high propensity to split during heat-treatment processes ${ }^{31,32}$ ) to investigate their shrinkage cracking behavior, which may be appropriately controlled by selecting a well-adjusted partial or bulk mesophase pitch derived from naphthalene. This enables the knowledge base of spinnable pitch precursors (not limited to isotropic pitches or bulk mesophase pitches) for preparing pitch-based carbon fibers with tunable microstructures and physical properties to be expanded and enriched.

\section{RESULTS AND DISCUSSION}

2.1. Unique Properties of the NP Precursor. In comparison with conventional complex pitch feeds, the NP precursor possesses distinctly unique features. The parent NP used as a carbonaceous feedstock has an SP of $124{ }^{\circ} \mathrm{C}$ and possesses a high TS fraction (CHS + CHI-TS) of $85.5 \mathrm{wt} \%$, a mid-range coking value $(\mathrm{CV})$, and a relatively low $\mathrm{AC}$ of 790 ppm (most of which is the residual $\mathrm{Al}_{2} \mathrm{O}_{3}$, and the heteroatoms, i.e., $\mathrm{N}$ and $\mathrm{S}$ are less than $100 \mathrm{ppm}$ ) as listed in Table 1. The AC in the NP can be further decreased to 170 ppm by simple solvent solubilization and filtration treatment so as to obtain an ultra-low-ash pitch (NP-TS) suitable for preparing a high-quality bulk mesophase pitch. The prepared NP has a relatively high SP and large average molecular weight $(\sim 500 \mathrm{~g} / \mathrm{mol})$ in contrast with a commercial petroleum pitch or coal-tar pitch (ca. $40-100{ }^{\circ} \mathrm{C}$ and $300-600 \mathrm{~g} / \mathrm{mol}$ measured by an identical test method), but the main molecular weight distribution $(300-800 \mathrm{~g} / \mathrm{mol})$ of the former is relatively narrow as shown in Figure $4 \mathrm{a}$. The high $\mathrm{H} / \mathrm{C}$ mol. ratio $(0.76)$ is a distinct characteristic of the NP precursor. It is well known that abundant hydrogen is beneficial to obtaining a spinnable pitch with a suitable SP (i.e., below $300{ }^{\circ} \mathrm{C}$ ) and good molten fluidity for melt-spinning. ${ }^{2,14}$ Furthermore, the NP precursor possesses good thermal stability below $300^{\circ} \mathrm{C}$ as shown in Figure 1 and an obvious exothermic peak initially appears at a relatively high temperature up to $\sim 431{ }^{\circ} \mathrm{C}$ due to 




Figure 1. Thermogravimetry-differential scanning calorimetry (TGDSC) curves of the NP precursor measured in a nitrogen atmosphere.

partial thermal pyrolysis of the NP (with a low weight loss of $\sim 30 \%$ ), which favors the controllable preparation of an LCcontaining pitch in an appropriate temperature range from 380 to $440{ }^{\circ} \mathrm{C}$ (the most preferred reaction temperature frequently used in this field). ${ }^{19,29}$

2.2. Characteristics of the SNPs with Representative LC Contents and Controllable SPs. Figure 2 shows the typical optical texture of the synthetic isotropic pitch (SIP) and low-mesophase-content pitch ( 5 vol \%) prepared by the airoxidation technique along with the SNPs with representative LC contents and the AR pitch. The various SNPs were prepared by thermal polycondensation at a reaction temperature of $420{ }^{\circ} \mathrm{C}$ (for Figure $2 \mathrm{c}-30$ vol \%) or $430{ }^{\circ} \mathrm{C}$ for $4-8 \mathrm{~h}$ (Figure $2 \mathrm{~d}-\mathrm{g}$ from 50 to $100 \mathrm{vol} \%$ ). The representative pitch samples selected have been pretreated by the nitrogenbubbling treatment for the purpose of subsequent meltspinning. It can be clearly seen that several NP-derived pitches with representative LC contents varying from 0 to $100 \mathrm{vol} \%$ can be readily obtained using suitable preparation methods and adjusting the thermal polymerization conditions. The SIP as shown in Figure 2a exhibits no optical activity (i.e., isotropy), when rotating the object stage of the polarized light microscope (PLM). The SNPs with a low content of LC (i.e., less than 30 vol \%) display optical anisotropy of sporadic mesophase spheres of various sizes interspersed in the continuous isotropic pitch matrix as shown in Figure $2 b, c$. As the LC content increases to $\sim 50$ vol \% (Figure $2 \mathrm{~d}$ ), large mesophase spheres and partial coalescence texture are present, and the isotropic pitch is still the continuous phase. On further increasing the LC content to $\sim 75$ vol $\%$, the optical texture changes into a coarse flow type, and some isotropic spherical or ellipsoidal domains are clearly mingled in the optically anisotropic continuous phase as shown in Figure 2e. The prepared SNP with a high LC content of $\sim 98$ vol \% exhibits a strongly oriented fine flow texture as shown in Figure $2 \mathrm{f}$. The MP-100 pitch thermally polymerized from the NP-TS fraction also displays a 100 vol \% LC content and an ideal oriented flow texture as shown in Figure $2 \mathrm{~g}$. The optical texture and liquid crystalline orientation of NP-derived pitches with high mesophase contents (e.g., 95, 98, and 100 vol \%) are very similar to that of the AR pitch as shown in Figure $2 \mathrm{~h} .{ }^{32}$

Table 2 lists the physical properties of the SIP- and NPderived pitches with various mesophase contents prepared by thermal polycondensation under various conditions. The NPderived pitches with representative $\mathrm{LC}$ contents ranging from 0 to $100 \mathrm{vol} \%$ possess SPs within a range of $210-290{ }^{\circ} \mathrm{C}$ that are controllable via the nitrogen-bubbling treatment, except for the 30 vol \% sample, which has a slightly low SP of $217^{\circ} \mathrm{C}$, because the M-30 pitch thermally polymerized in a nitrogen atmosphere possesses a relatively low polycondensation degree compared with that of the M-5 pitch prepared by an airblowing technique. These NP-derived mesophase-containing pitches all possess excellent characteristics, such as suitable SPs, relatively high $\mathrm{H} / \mathrm{C}$ mol. ratios, and large CVs compared with those of polyacrylonitrile or rayon, ${ }^{12,16}$ and acceptable ACs (less than 0.15 wt \%, i.e., the minimum $\mathrm{AC}$ value for a commercially available high-softening-point pitch). The AC in the SNPs increases markedly with the increase of mesophase contents due to the gradual improvement of the polycondensation degree of pitch melt. The volatilization of lowmolecular-weight components results in the final concentration of $\mathrm{AC}$ in the heavy residue, i.e., pitch product. These characteristics of NP-derived pitches are similar to some extent to those of the AR pitch and make them good pitch precursors for preparing pitch-based carbon fibers.

The ${ }^{1} \mathrm{H}$ NMR spectra of the soluble fractions from the NP precursor and its derived SIP and SNPs with two levels of LC content are shown in Figure 3. According to the wellestablished Brown-Ladner procedure ${ }^{35}$ and previous similar work, ${ }^{37,38}$ hydrogen located at a chemical shift of 6-10 or 4.5$6 \mathrm{ppm}$ in the ${ }^{1} \mathrm{H}$ NMR spectrum is assigned to aromatic hydrogen or alkene hydrogen (i.e., hydrogen on a carboncarbon double bond), hydrogen distributed at a chemical shift of $2-4.5,1.1-2$, and $0.3-1.1 \mathrm{ppm}$ is ascribed to $\alpha, \beta$, and $\gamma$ aliphatic hydrogen, respectively, and the medium-strength peak at a chemical shift of ca. $1.35 \mathrm{ppm}$ is attributed to the naphthenic $-\mathrm{CH}_{2}-$. As can be seen from Figure $3 a-d$, although the variety of aromatic hydrogen types for the SIP and the SNPs reflects the various degrees of polycondensation, the peak intensity for aromatic hydrogen in the NP precursor obviously decreases after the thermal polycondensation reaction. According to the hydrogen distribution calculated by ${ }^{1} \mathrm{H}$ NMR analysis as listed in the Supporting Information (Table S1), the contents of aromatic hydrogen and $\gamma$ aliphatic hydrogen in the NP precursor are relatively high and low, respectively, compared to other forms of hydrogen, which indicates that the typical aliphatic side chain in the NP is very short, possibly in the form of methyl and ethyl groups. ${ }^{2,15}$ After the thermal polycondensation reaction under various conditions, the content of aromatic hydrogen has significantly reduced, but the contents of alkene hydrogen and aliphatic hydrogen at different substitution sites increase to various degrees. The large increase in $\beta$ aliphatic hydrogen indicates that the naphthenic structure is abundant in the NP-derived synthetic pitches. It can be observed that the content of $\beta$ aliphatic hydrogen clearly increases with the increase of the anisotropic mesophase content in the SNPs, which indicates that a large amount of naphthenic structure forms during the thermal polymerization of the NP. This indicates that largemolecular-weight aromatic hydrocarbons with a planar structure can be formed from a number of naphthenic structures and $-\mathrm{CH}_{2}-$ bridge-linking of some naphthalenederived oligomers. ${ }^{2,15}$

The mass spectra of the NP and its derived SIP and SNPs with two levels of LC content are shown in Figure 4. It can be seen that the molecular weight distribution of the NP ranges 

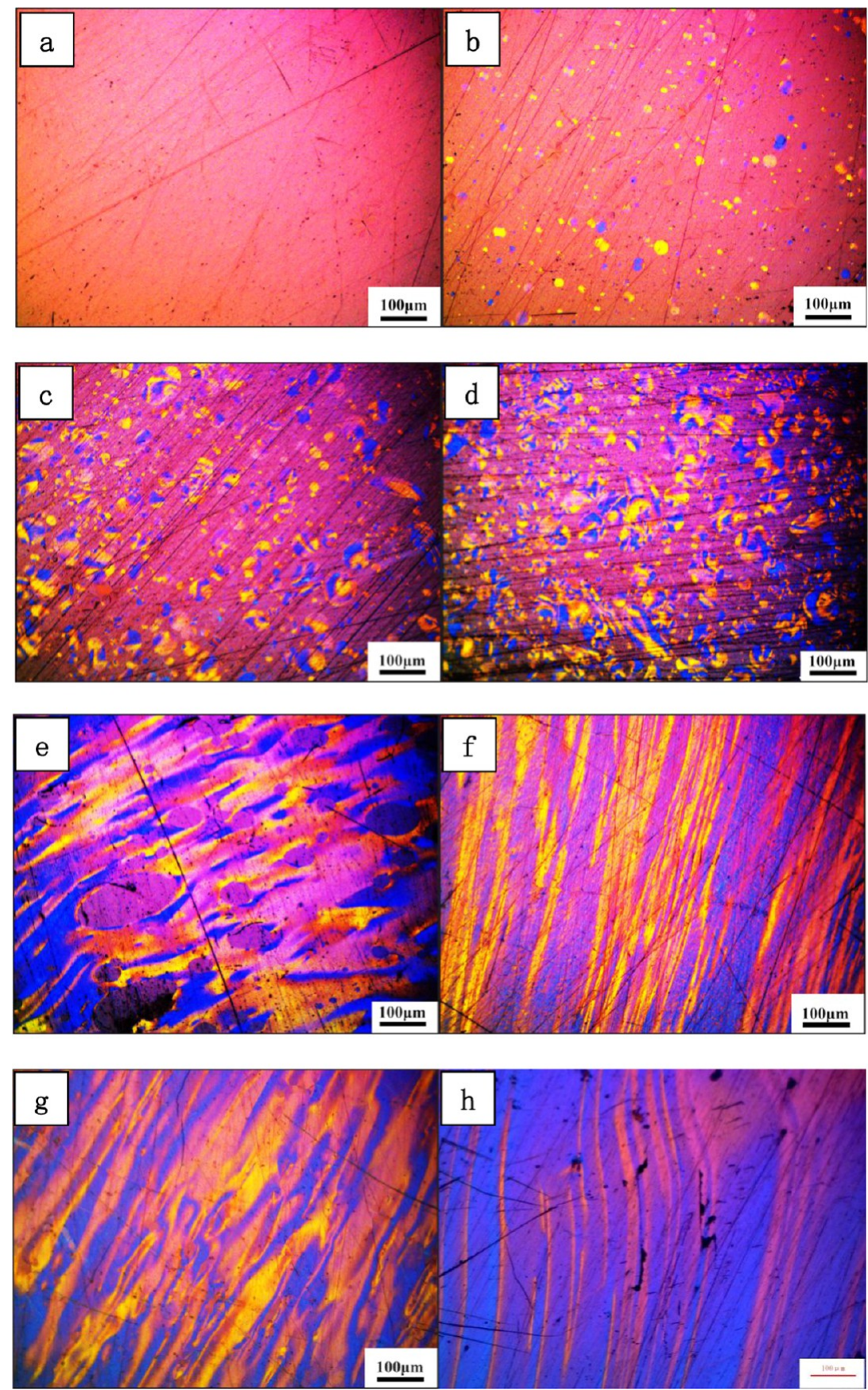

Figure 2. Optical texture of the SIP (a) and low-mesophase-content ( $5 \mathrm{vol} \%)$ pitch (b) prepared by the air-oxidation technique, and the various NP-derived pitches prepared by direct thermal polycondensation showing representative LC contents (c, 30\%; d, 50\%; e, 75\%; f, 98\%; and g, 100 vol \%) as well as the AR pitch (h).

from 200 to $800 \mathrm{~m} / z$ and the main peak appears at $\sim 500 \mathrm{~m} / z$ as shown in Figure 4a, which indicates that the main component of the NP precursor is a naphthalene tetramer. After air-oxidation treatment, the molecular weight distribution of the SIP has increased greatly to between 800 and $4000 \mathrm{~m} / z$, and the most abundant molecular weight is around $2000 \mathrm{~m} / \mathrm{z}$ as shown in Figure $4 b$, which indicates that the oligomers in the NP precursor are extensively polymerized due to the role of thermal oxidation. The most abundant molecular weights of the SNPs with two levels of LC content (50\% and 98 vol \%) are concentrated at $\sim 2200$ and $\sim 2600 \mathrm{~m} / z$, respectively, as shown in Figure 4c,d and the molecular weight distribution of the 98 vol \% mesophase pitch is relatively narrow. This indicates that the ca. $100 \mathrm{vol} \%$ naphthalene-based mesophase pitch is a medium-weight polymer with an average molecular weight of $\sim 2600 \mathrm{~g} / \mathrm{mol}$, consisting of large mesogen units (a ladder-shaped molecular structure) formed by 20 naphthalene molecules through thermally induced aromatic growth. $^{2,15,39,40}$ It is interesting to note that there is no obvious difference in the molecular weight characteristics for the two types of pitches (i.e., the SIP and mesophase pitch M50) when they have a similar SP. However, the molecular morphology, configuration, and arrangement in the mesophase pitch (i.e., its capability to form a nematic liquid crystalline structure) result in a variety of useful physical properties and promising applications. 
Table 2. Physical Properties of the SIP- and NP-Derived Pitches with a Range of Mesophase Contents Prepared by Thermal Polycondensation Under Various Conditions

\begin{tabular}{|c|c|c|c|c|c|c|c|c|}
\hline \multirow[b]{2}{*}{ sample $^{a}$} & \multicolumn{3}{|c|}{ main fraction/wt $\%$} & \multirow[b]{2}{*}{$\mathrm{SP} /{ }^{\circ} \mathrm{C}$} & \multirow[b]{2}{*}{ yield/wt \% } & \multirow[b]{2}{*}{$\mathrm{CV} /$ wt \% } & \multirow[b]{2}{*}{$\mathrm{AC} / \mathrm{ppm}$} & \multirow[b]{2}{*}{$\mathrm{H} / \mathrm{C}(\mathrm{mol})$} \\
\hline & $\mathrm{TS}$ & TI-QS & QI & & & & & \\
\hline SIP & 21.8 & 47.6 & 30.6 & 239 & 70.4 & 69.3 & 1080 & 0.59 \\
\hline M-5 & 23.8 & 43.5 & 32.7 & 241 & 68.8 & 70.4 & 1090 & 0.58 \\
\hline M-30 & 29.4 & 33.7 & 36.9 & 217 & 74.5 & 73.2 & 1020 & 0.57 \\
\hline M-50 & 23.3 & 36.6 & 40.1 & 243 & 71.3 & 78.3 & 1150 & 0.55 \\
\hline M-75 & 14.8 & 24.5 & 60.7 & 257 & 60.8 & 85.1 & 1340 & 0.54 \\
\hline M-98 & 11.0 & 14.8 & 74.2 & 282 & 55.3 & 90.5 & 1480 & 0.52 \\
\hline $\mathrm{MP}-100^{b}$ & 9.3 & 15.1 & 75.6 & 285 & 55.0 & 91.0 & 290 & 0.51 \\
\hline AR pitch & 23.1 & 26.1 & 50.8 & 265 & & 78.6 & 30 & 0.56 \\
\hline
\end{tabular}

${ }^{a}$ The prepared SNP sample was labeled "M- $x$ ", where $x$ is the relevant mesophase content $\% .{ }^{b} \mathrm{MP}-100$ is the 100 vol $\%$ mesophase pitch made from the TS fraction of the NP.
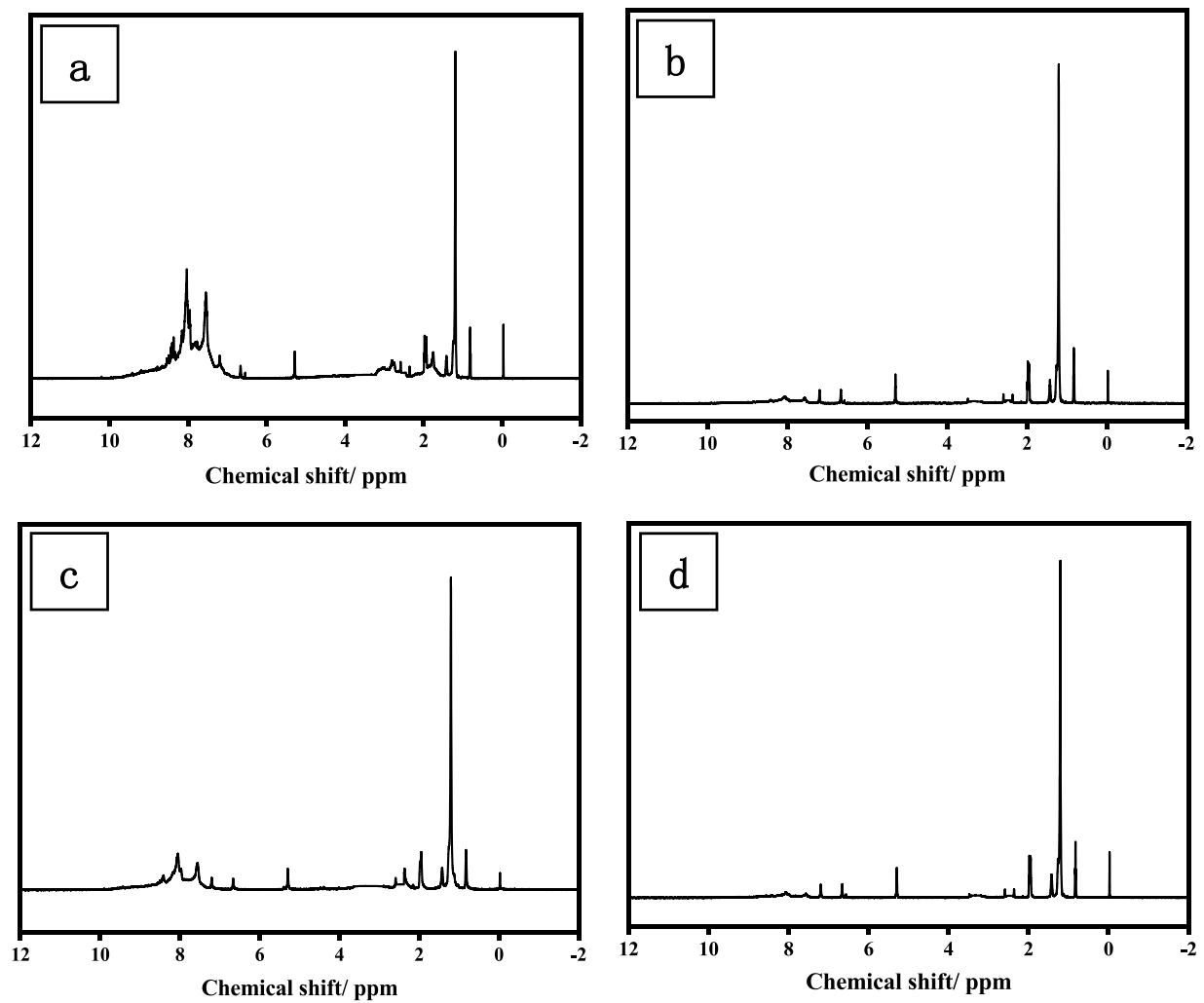

Figure 3. ${ }^{1} \mathrm{H}$ NMR spectra of the soluble fractions from the NP (a) and its derived SIP (b) and SNPs with two levels of LC content (c-50\% and d98 vol \%).

2.3. Phase Separation, Thermal Stability, and MeltSpinnability of the SNPs with Representative LC Contents. An SNP with an LC content of $\sim 40$ vol \%, prepared by thermal polymerization without the nitrogenbubbling treatment, was selected for investigation of the phaseseparation behavior of LC in the as-prepared SNP. It can be clearly seen that the LC in the SNP exhibits obvious phase separation of the isotropic and anisotropic components, as shown in Figure 5, after allowing the SNP to stand for $6 \mathrm{~h}$ in a vertical test tube at a temperature of $\sim 290{ }^{\circ} \mathrm{C}$ (i.e., $\sim 80{ }^{\circ} \mathrm{C}$ above its SP of $\sim 210^{\circ} \mathrm{C}$ ). It is remarkable that the $\mathrm{LC}$ appears to exhibit complicated phase conversion behavior of mutual cohesion, coalescence, and self-organization during the thermal soaking process. The cause of liquid crystalline phase separation is the slight difference in density between the two phases when heat-soaked at a suitable temperature for a long time since heating results in a low-viscosity system owing to the significant presence of light fractions ( $28 \mathrm{wt} \% \mathrm{TS})$ in the as-prepared SNP.

In contrast, when the nitrogen-bubbling treatment was applied to remove some of the light fractions, the isotropic and anisotropic components in the synthetic pitches with various mesophase contents form a relatively stable system and no obvious phase separation occurs, as shown in the Supporting Information (Figure S1), after allowing the SNPs to stand for 6 $\mathrm{h}$ in a vertical test tube at the corresponding melt-spinning temperature. It can be clearly seen that the optical textures at various locations (i.e., top, middle, and bottom) in the tube show no obvious differences, except for a slight textural difference of prepared SNPs, as shown in Figure 2, owing to the thermal movement and molecular assembly of LC during the long soaking treatment. ${ }^{20,25,32}$ This indicates that the 

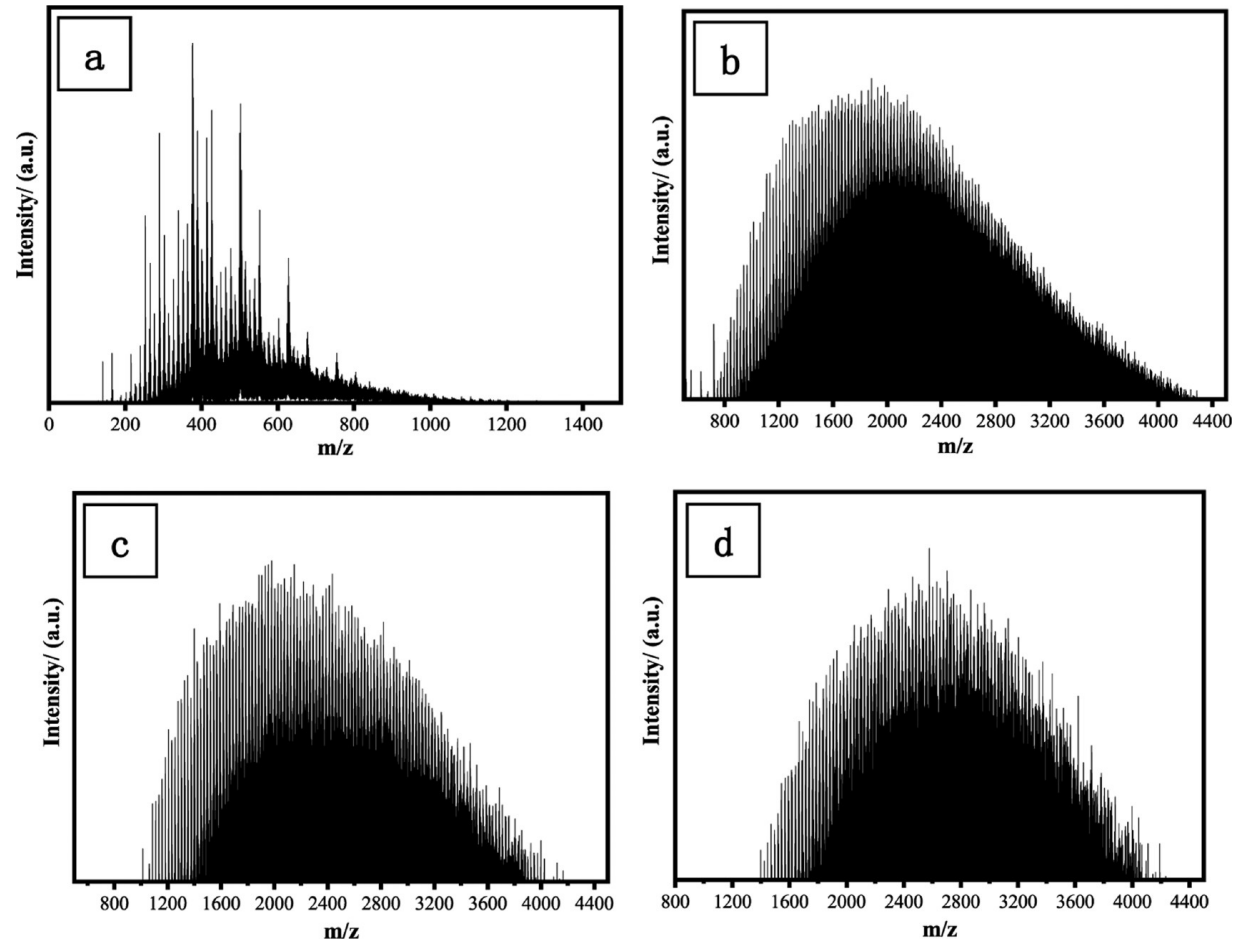

Figure 4. Mass spectra of the NP (a) and its derived SIP (b) and SNPs with two levels of LC content (c-50\% and d-98 vol \%).

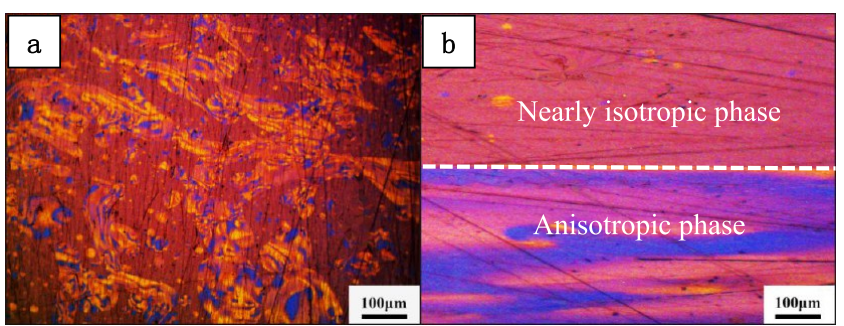

Figure 5. Optical texture of SNP with an LC content of $\sim 40$ vol $\%$ (a) before and (b) after allowing it to stand for $6 \mathrm{~h}$ in a vertical test tube at a temperature $\sim 80{ }^{\circ} \mathrm{C}$ above its SP.

thermal stability and phase separation of the SNPs with various liquid crystalline contents were successfully controlled by the nitrogen-bubbling treatment.

The thermal stability of the SNPs with LC contents ranging from $5 \%$ to 98 vol \% after the nitrogen-bubbling treatment is further verified by the TG analyses as shown in Figure 6. In comparison to the NP precursor, the various thermally polymerized SNPs possess a high $\mathrm{CV}$ of above 70 wt \% that increases with the LC content and good thermal stability at high temperatures up to $380{ }^{\circ} \mathrm{C}$ without any obvious pyrolysis. This implies that the SNPs would be very stable during the whole period $(3-4 \mathrm{~h})$ required for melt-spinning within the commonly used temperature range of $320-360{ }^{\circ} \mathrm{C}$.

The molten flow distance-temperature curves of the NPderived synthetic pitches with various LC contents before and after the nitrogen-bubbling treatment are shown in Figure S2. It can be clearly seen that the gradual softening and plastic flow behaviors of the representative synthetic pitches (ranging from isotropic pitch, i.e., the SIP to bulk mesophase pitch, i.e., MP100) are all quite similar. Once the pitch is softened to a certain degree (corresponding to the initial sloping line in each curve) and the flow of pitch becomes very facile (corresponding to the final steeply sloping part of each curve). This good

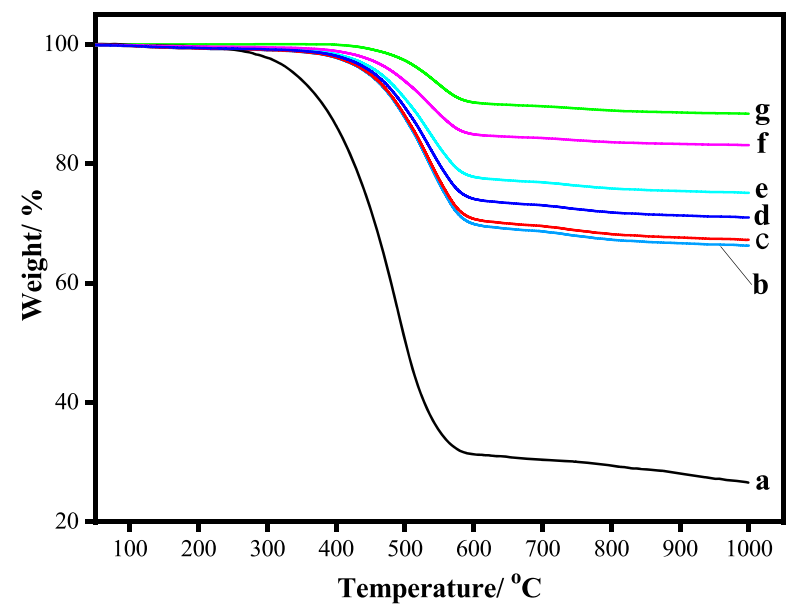

Figure 6. TG curves of the NP precursor (Line a) and its derived SIP (Line b) and SNPs with various LC contents (line c, 5\%; line d, 30\%; line e, 50\%; line f, 75\%; and line g, 98 vol \%).

molten fluidity is very favorable for pitch melt-spinning. Increasing the LC content in the pitches increases the temperatures for the onset of each intermediate zone, which corresponds to the SPs of the pitch samples. The unblown lowmesophase-containing pitch ( $\sim 5$ vol \%) shows a very low SP (slightly higher than that of the NP (not shown)), which further corroborates the idea that the nitrogen-bubbling pretreatment effectively removes the light fractions in the pitch and thereby increases the SP of a pitch so as to render it spinnable.

The melt-spinnability of the thermally polymerized SIP and the NP-derived pitches with LC contents varying from $5 \%$ to 100 vol \% along with the standard reference AR pitch is listed in Table S2. It can be seen that both the SIP and SNPs with a high LC content (larger than $\sim 50$ vol \%) possess good 
spinnability based primarily on the continuity of melt-spinning without a filament break and the homogeneity of fiber diameter. The more the anisotropic content the SNP possesses, the more homogeneously the pitch behaves, and the better the spinnability of the pitch. The SNPs with a low LC content (less than $\sim 30$ vol \%) show poor spinnability owing to the heterogeneity of their components and their spatial distributions, as shown in Figures 2 and S1. Some liquid crystalline spheres with different sizes mingled in the isotropic pitch matrix may introduce internal stress concentrations in the fragile pitch fiber and result in frequent breaks. The resulting discontinuous and nonuniform pitch fibers are not appropriate for the subsequent comparative research. It is worth noting that the SNP with a mesophase content of 98 vol \% and a relatively high AC of $1480 \mathrm{ppm}$, and the NP-derived MP-100 pitch with a $100 \mathrm{vol} \%$ mesophase content and a relatively low AC of 290 ppm both exhibit very good spinnability, similar to that of the AR mesophase pitch with an ultralow AC of 30 ppm. This indicates that except in the case of the SIP, the spinnability is not dominated by the low AC (as long as it is less than 0.2 wt \%) but by the liquid crystalline texture (i.e., mesophase content and liquid crystalline orientation) and homogeneity of phase distribution in the pitch precursor, which has never been reported in previous work.

On the basis of the thermal stability, molten fluidity, and melt-spinnability of various spinnable pitches derived from the NP precursor discussed above, an experimental phase diagram is shown in Figure 7. This shows spinnability as a function of

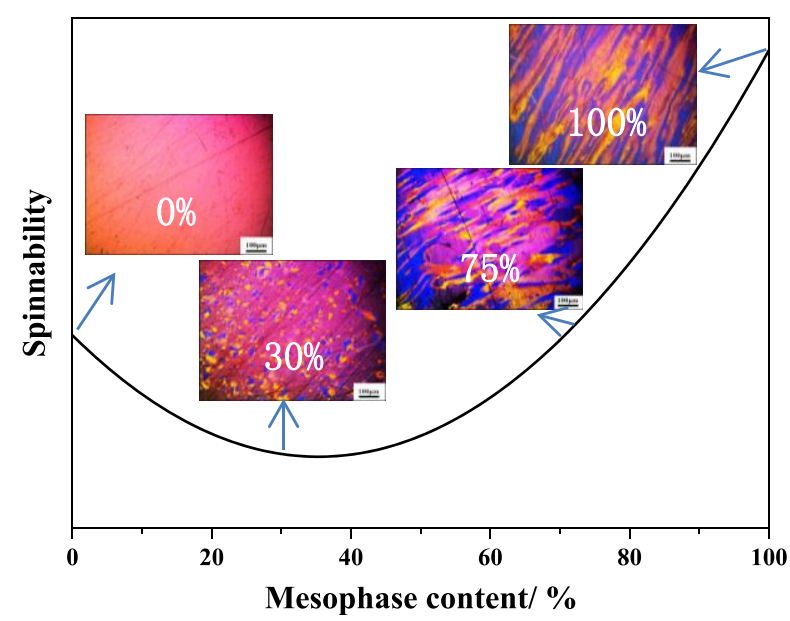

Figure 7. Phase diagram of spinnability vs. mesophase content as measured for the NP-derived two-phase spinnable pitches with SPs in the range of $210-290{ }^{\circ} \mathrm{C}$. The inserted images are the optical textures of representative mesophase contents.

mesophase content for NP-derived two-phase spinnable pitches with SPs in the range of $210-290{ }^{\circ} \mathrm{C}$ and can be used to predict the spinnability of a synthetic pitch according to the mesophase content or act as a reference for partial mesophase pitches derived from other carbonaceous feedstocks. The result is generally consistent with previous research that blended a maximum content of $30 \mathrm{wt} \%$ isotropic pitch in a mesophase pitch to form a homogeneous continuous phase for improved melt-spinning. ${ }^{30}$ Similarly, it is not unexpected that the SNP-30 sample with 30 wt \% mesophase in its isotropic pitch matrix displays the worst spinnability. This new phase diagram for the mesophase-containing pitches (ranging from the isotropic pitch to the bulk mesophase pitch) is proposed here to offer a straightforward guide for the preparation of pitch-based carbon fibers.

2.4. Morphology, Microstructures, and Physical Properties of the Derivative Large-Diameter Carbon

Fibers. Typical optical textures of the transversal sections of carbon fibers derived from the SIP and SNPs with various LC contents are shown in Figure S3. It can be clearly seen that the pitch fibers made from the SIP have a uniform diameter and no optical anisotropy as shown in Figure S3a. Liquid crystalline spheres are dispersed in the cross sections of carbon fibers derived from a low-mesophase-content SNP ( $\sim 5$ vol \%) as shown in Figure S3b, and the fiber diameters are nonuniform owing to the poor spinnability of the pitch. The optical textures of the carbon fibers made from the SNPs with three representative LC contents (50, 75\%, and 98 vol \%) as shown in Figure $\mathrm{S} 3 \mathrm{c}-\mathrm{e}$ display obvious differences in the distribution and size of isochromatic color regions (which are closely related to the orientation and size of microcrystalline domains within the carbon fibers). Thus, the arrangement, orientation, and distribution of LC in these carbon fibers are different, being inherited from the texture of the precursor pitch. The degree of splitting (i.e., the abundance of cracked fibers and the angle of the resulting wedge-shaped void in a single fiber) is generally greater in the carbon fibers made from a pitch with a high mesophase content. The more severe cracking with increasing mesophase content in the pitch precursor is in accordance with previous research..$^{31-33}$ However, it is interesting, and in the latter case surprising, to note that no splitting is observed in the cross sections of carbon fibers derived from the SNPs with $\sim 50 \%$ and 100 vol \% mesophase contents (i.e., SNP-50 and MP-100) as shown in Figure S3c,f. This means that the common cracking behavior in mesophase pitch-based carbon fibers could be avoided by selecting a partial or bulk mesophase pitch derived from naphthalene.

Typical scanning electron microscopy (SEM) images of surface and transversal sections of carbon fibers derived from the SIP and SNPs with various LC contents after heat treatment at $1000{ }^{\circ} \mathrm{C}$ are shown in Figure 8. The carbon fibers made from the SIP are uniform in diameter, and their crystallite size and orientation are relatively low, which indicates low crystallinity (in terms of qualitative comparison) as shown in Figure $8 a_{1}-a_{3}$, and there are no open cracks in their cross sections. The diameter of the carbon fibers derived from the SNP with a low mesophase content $(\sim 5$ vol \%) is nonuniform, and the fiber surface is uneven as shown in Figure $8 b_{1}-b_{3}$ owing to the presence of dispersed carbon microspheres derived from the spherical liquid crystals in the pitch precursor. There is an obvious difference in the volume shrinkages of the isotropic and anisotropic components in the pitch fibers. The carbon fibers made from the SNP with a medium mesophase content ( $\sim 50 \mathrm{vol} \%)$ possess a uniform diameter, a smooth surface, and identical crystallinity as shown in Figure $8 c_{1}-c_{3}$. These carbon fibers exhibit typical random textures (small crystallite size and disordered crystallite arrangement) and show no shrinkage cracking during the heat-treatment processes. A clear correlation between the transversal texture of carbon fibers and their crystallite size has been reported in previous work. ${ }^{32}$ Obvious open cracks appeared in the carbon fibers prepared from the SNPs with a high mesophase content (75\% and 98 vol \%) as shown in Figure $8 \mathrm{~d}_{2}, \mathrm{e}_{2}$. A typical "Pac-man" splitting texture (in the cross section of large-diameter fibers, carbon layers radiate from the center to the surface like spokes in a wheel) can be 

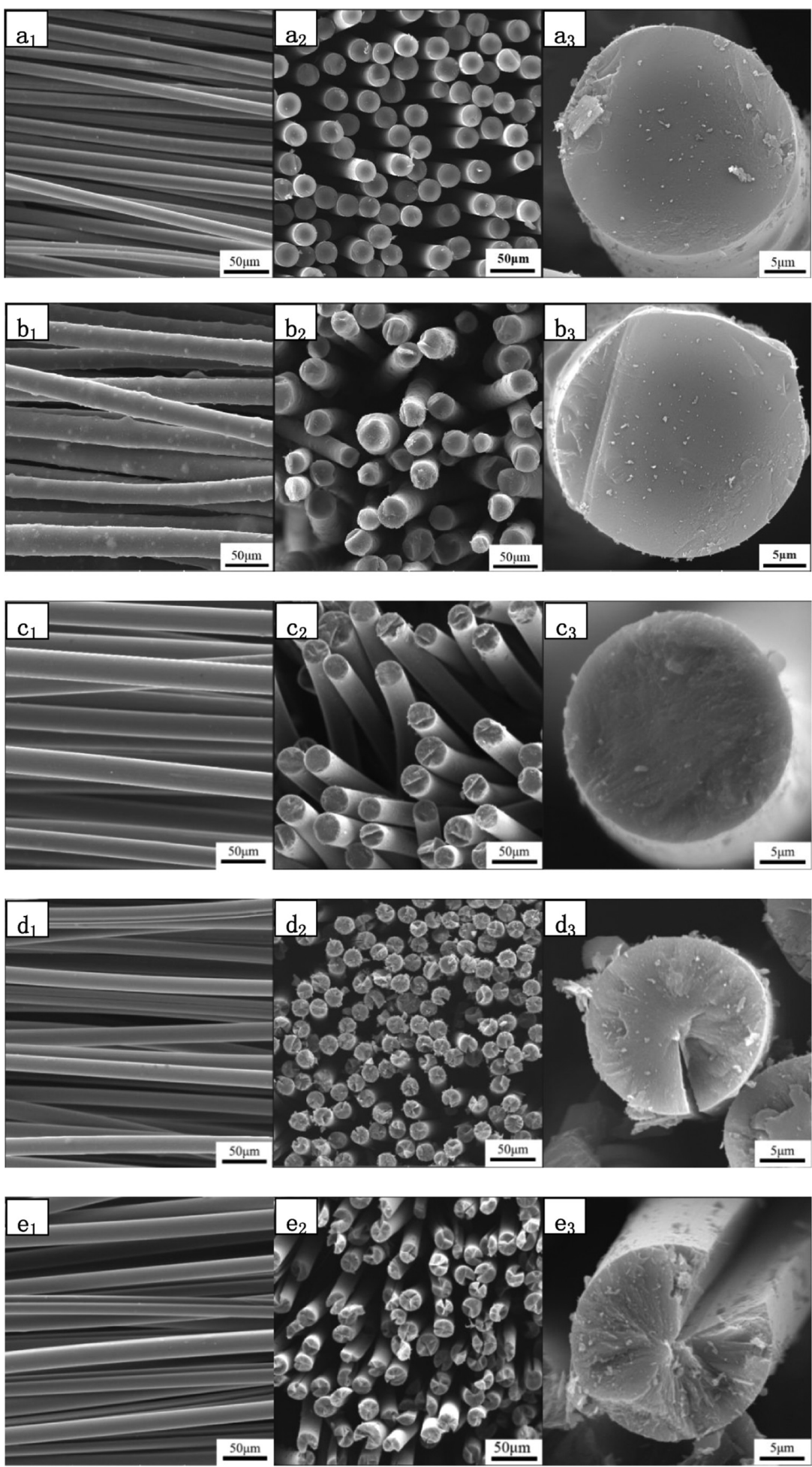

Figure 8. Typical SEM micrographs of carbon fibers derived from the SIP (a) and SNPs with various LC contents (b, 5\%; c, 50\%; d, 75\%; and e, 98 vol \%) after heat treatment at $1000{ }^{\circ} \mathrm{C}$ (subscripts 1,2 , and 3 denote the fiber surface, fiber transversal section, and magnified fiber cross section, respectively).

seen clearly in Figure $8 \mathrm{~d}_{3}, \mathrm{e}_{3}$. This splitting is similar to that observed in the well-known K-1100 carbon fibers ${ }^{33,41}$ and in previous work. ${ }^{31-33}$ The degree of splitting in the carbon fibers is obviously aggravated with the increase of the LC content in the precursor pitches from $75 \%$ to 98 vol \%, and originally stems from the significant improvement of the textural orientation of LCs in the pitch precursors. This is in good agreement with the PLM analysis results shown in Figure 2.

Figure 9 shows the cross-sectional SEM micrographs of carbon fibers derived from the two types of 100 vol \% mesophase pitches with a low AC (i.e., MP-100 and AR pitch) after heat treatment at $1000{ }^{\circ} \mathrm{C}$. It is surprising to note that there are no open cracks in the carbon fibers with a radial 

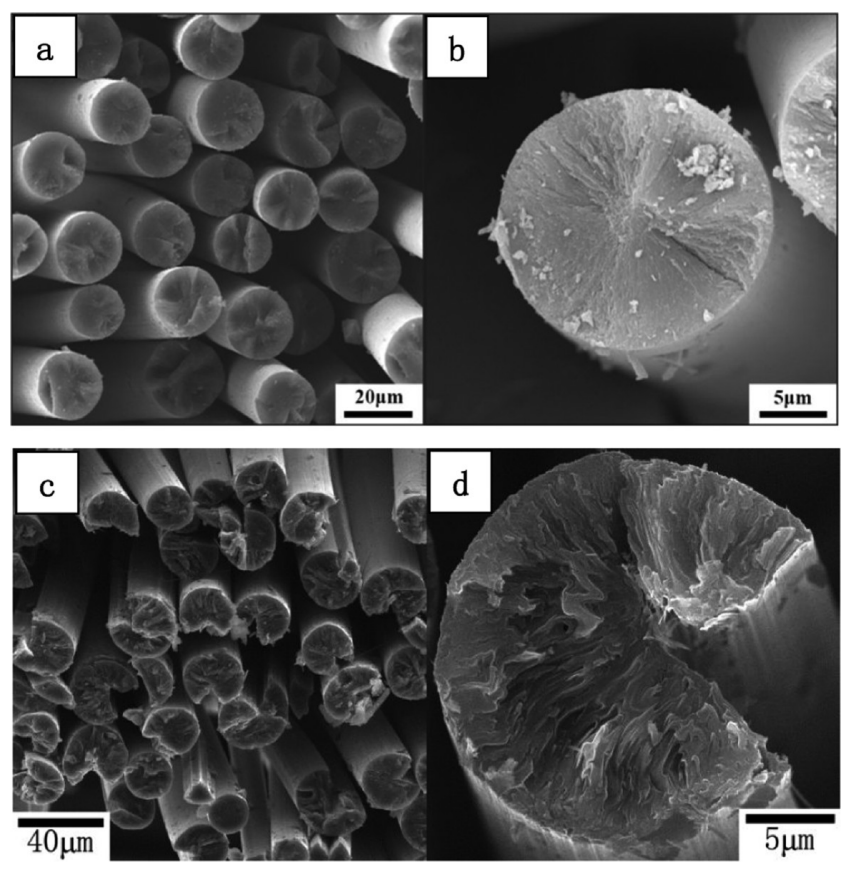

Figure 9. Cross-sectional SEM micrographs of carbon fibers derived from the two types of 100 vol \% mesophase pitches ( $a$ and $b$ from $\mathrm{MP}-100, \mathrm{c}$ and $\mathrm{d}$ from the AR pitch) after heat treatment at $1000{ }^{\circ} \mathrm{C}$.

texture derived from the MP-100 pitch with a 100 vol \% mesophase content as shown in Figure 9a,b. This is completely different from what is seen for the SNP pitch-derived carbon fibers as shown in Figure $8 \mathrm{e}$ and $\mathrm{AR}$ pitch-prepared carbon fibers as shown in Figure 9c,d. The absence of cracks in the MP-100 pitch-derived fibers may be traced back to the starting feedstock, i.e., the TS fraction of the NP precursor (which possesses a highly uniform molecular weight and medium molecular plane size by the removal of some large aromatic molecules, i.e., the TI fraction in the NP). This can be developed into carbon fibers with a medium preferred crystallite orientation in the subsequent heat treatment, and this may inhibit crack propagation. In contrast, AR pitchderived carbon fibers display a good crystallite orientation and large graphenelike carbon layers notwithstanding the presence of severe shrinkage cracking as indicated in previous work. ${ }^{32,33}$

$\mathrm{X}$-ray diffraction (XRD) patterns of several carbon fibers derived from the SIP and SNPs with various LC contents (0$100 \mathrm{vol} \%$ ) after heat treatment at $1000{ }^{\circ} \mathrm{C}$ are shown in Figure S4. There is a similar pattern of one broad diffraction peak at around $2 \theta=23^{\circ}$ corresponding to the $\left(\begin{array}{lll}0 & 0 & 2\end{array}\right)$ crystal plane of hexagonal graphite. The relative intensity of $\left(\begin{array}{lll}0 & 0 & 2\end{array}\right)$ diffraction peaks from the various carbon fibers seems to increase gradually with increasing LC content in the pitch precursor, and the $\left(\begin{array}{lll}0 & 0 & 2\end{array}\right)$ diffraction peaks have obviously shifted to a higher $(2 \theta)$ angle, which is characteristic of increasing graphitization. ${ }^{1,2}$ The microcrystalline parameters of the various carbon fibers are listed in Table S3. It can be seen that, with increasing mesophase content in the precursor pitch, the interlayer spacing $\left(d_{002}\right)$ in the carbon fibers obviously decreases, and the crystallite stack height $\operatorname{Lc}_{(002)}$ and the crystallite coherence length $\mathrm{La}_{(100)}$ in the carbon fibers obviously increase. $^{42}$ The SIP-derived carbon fibers show a large $d_{002}$ and small crystallite size, similar to those of generalgrade isotropic-pitch-based carbon fibers, which are nongraphitizable. ${ }^{1}$ There is no great difference between the crystallite sizes for the carbon fibers made from high-content mesophase pitches (e.g., $98 \%$ and $100 \mathrm{vol} \%$ ), except that the AR pitch-derived carbon fibers possess a relatively low $d_{002}$, implying that it may easily form a graphitic carbon in subsequent graphitization treatment. ${ }^{32}$ The XRD analysis results are highly consistent with the optical texture and microstructure analyses as shown in Figure S3 and Figure 8.

The tensile strength, Young's modulus, and axial electrical properties of carbon fibers derived from the various spinnable pitches after heat treatment at $1000{ }^{\circ} \mathrm{C}$ are listed in Table 3 . It can be clearly seen that the tensile strength and Young's modulus of the carbon fibers significantly increase with increasing mesophase content in the precursor pitch (except for the carbon fibers made from the 5 vol \% mesophase content pitch due to their poor spinnability and heterogeneous structure). In addition, the elongation at break and electrical resistivities of the same series of carbon fibers exhibit a gradually decreasing trend, which is the direct result of the microcrystalline size, crystallite orientation, and ordered stacking of graphenelike layers within the carbon fibers as shown in Figures 8 and 9. Precursor pitch with a high mesophase content and a good textural orientation therefore results in the anticipated significant increases in the mechanical properties ( $\sigma$ and E) of the carbon fibers, in accordance with previous studies. $8,32,33,43$ The AC in the precursor pitch has an obvious effect on the electrical resistivity of the resultant carbon fibers derived from the two bulk mesophase pitches with a similar textural orientation (i.e., MP-100 and AR pitch) because the higher $\mathrm{AC}$ remaining in the MP-100-derived carbon fibers will finally impede the mobilization or conduction of free electrons smoothly on the carbon planes ${ }^{8}$ and increase the electrical resistivity, as can be seen in Table 3. The elongation at break $(\sim 1 \%)$ and Young's modulus $(45$ $\mathrm{GPa})$ of the SIP-derived carbon fibers are, respectively, smaller and larger than those of commercial isotropic-pitch-based carbon fibers $\left(2.0-2.5 \%\right.$ and $30-40 \mathrm{GPa},{ }^{5}$ not shown in the

Table 3. Mechanical and Electrical Properties of Carbon Fibers with a Large Diameter of $\sim 20 \mu \mathrm{m}$ Derived from Various Spinnable Pitches after Heat Treatment at $1000{ }^{\circ} \mathrm{C}$

\begin{tabular}{lcccc}
\hline \multicolumn{1}{c}{ sample } & tensile strength $\sigma / \mathrm{MPa}$ & elongation at break $\varepsilon / \%$ & Young's modulus E/GPa & electrical resistivity/ $\mu \Omega \mathrm{m}$ \\
CF-SIP & $440 \pm 25$ & $0.98 \pm 0.05$ & $45 \pm 3$ & $52.4 \pm 1.8$ \\
CF-SNP-5 & $280 \pm 22$ & $0.94 \pm 0.06$ & $30 \pm 3$ & $53.6 \pm 2.2$ \\
CF-SNP-50 & $540 \pm 35$ & $0.82 \pm 0.05$ & $66 \pm 5$ & $34.4 \pm 1.1$ \\
CF-SNP-75 & $680 \pm 42$ & $0.76 \pm 0.04$ & $90 \pm 6$ & $29.2 \pm 0.9$ \\
CF-SNP-98 & $840 \pm 50$ & $0.75 \pm 0.04$ & $112 \pm 7$ & $26.5 \pm 0.8$ \\
CF-MP-100 & $950 \pm 58$ & $0.75 \pm 0.04$ & $127 \pm 8$ & $25.9 \pm 0.7$ \\
CF-AR & $980 \pm 60$ & $0.74 \pm 0.03$ & $132 \pm 8$ & $17.4 \pm 0.5$
\end{tabular}


table), and this indicates that the NP-derived isotropic carbon fibers exhibit good stiffness.

It is interesting to note that the tensile strengths of largediameter carbon fibers treated at $1000{ }^{\circ} \mathrm{C}$, prepared from SNPs with $98 \%$ and $100 \mathrm{vol} \%$ mesophase contents, are relatively high at about 840 and $950 \mathrm{MPa}$, respectively, despite their relatively high ACs (1480 and $290 \mathrm{ppm}$ ). These tensile strengths are comparable to that of ultra-low-AC (30 ppm) AR-pitch-based carbon fibers $(980 \mathrm{MPa})$. Although the mechanical properties of these large-diameter $(\sim 20 \mu \mathrm{m})$ carbon fibers are lower than those of commercial mesophasepitch-based carbon fibers with a small diameter of $\sim 10 \mu \mathrm{m},{ }^{6,7}$ their axial electrical and thermal conduction properties ${ }^{32}$ as well as cost-competitive production will be promising. It can be seen that there is no obvious difference in the tensile strength and Young's modulus of the carbon fibers derived from the two types of $100 \mathrm{vol} \%$ mesophase pitches (MP-100 and AR pitch) in spite of an obvious difference in the AC of the two. ${ }^{32}$ The differences in the $\mathrm{AC}$ in the pitch precursors seem to have less effect on the mechanical properties of $1000{ }^{\circ} \mathrm{C}$ carbonized large-diameter (e.g., $\Phi \sim 20 \mu \mathrm{m}$ ) fibers, than for smaller fibers (e.g., $\Phi \sim 10 \mu \mathrm{m})$, a fact that is rarely mentioned in previous work on carbon fibers, except for the references to $\mathrm{AC}$ in highly purified pitch-derived carbon fibers. ${ }^{2,15,44}$

\section{CONCLUSIONS}

This work clearly demonstrates the feasibility of a controllable preparation of SNPs with various LC contents derived from the unique NP precursor and the consummate bridge connecting isotropic-pitch-based and anisotropic mesophasepitch-based carbon fibers. Nitrogen-bubbling treatment of the prepared SNPs is a simple and effective method to adjust the constituents, chemical structure, and physical properties of these two-phase spinnable pitches and to improve their thermal stability as well as to control the phase-separation behavior of LC in the SNPs, which facilitates the meltspinnability of two-phase coexisting pitches. Melt-spinnable SNPs with representative mesophase contents $(5,50,75,98 \%$, and 100 vol \%) and suitable SPs ranging from 220 to $290{ }^{\circ} \mathrm{C}$ can be utilized to prepare large-diameter carbon fibers with a variety of morphologies, microstructures, and physical properties for various promising applications.

An experimental phase diagram of the spinnability and mesophase content, as measured for the NP-derived two-phase spinnable pitches with SPs in the range of $210-290{ }^{\circ} \mathrm{C}$, is newly proposed in this work for predicting the spinnability of a mesophase-containing pitch for preparing pitch-based carbon fibers. The LC contents (optical texture and spatial distribution) in the SNPs have an important influence on the constituents, molecular structure, and physical properties of the various spinnable pitches. This ultimately dictates the morphology (transversal texture and degree of fiber splitting) and microstructure (crystallite size and preferred orientation) of the resulting carbon fibers, which in turn strongly influence the final physical properties of the fibers. It is interesting to note that the relatively high AC (albeit still less than $0.15 \mathrm{wt}$ $\%)$ in the well-adjusted mesophase pitch precursor seems to have no obvious effect on the pitch spinnability and the mechanical properties of large-diameter carbon fibers, and this phenomenon has never before been reported in previous work. The Pac-man splitting phenomenon commonly observed to occur during heat treatment of carbon fibers derived from high vol \% mesophase pitch can be effectively limited or even avoided by suitable preprocessing to tailor the composition of a partial or bulk mesophase pitch. This remarkable finding can consequently promote the wide application of tailorable mesophase-containing naphthalene-based pitches with an achievable and acceptable AC (300-1500 ppm) catalytically synthesized by $\mathrm{AlCl}_{3}$ and thereby pave the way for reducedcost and high-performance pitch-based carbon fibers.

\section{EXPERIMENTAL SECTION}

4.1. Preparation of Various SNPs with Isotropy and Anisotropy. A kilogram-scale batch of NP synthesized in the laboratory by catalytically polymerizing pure naphthalene with anhydrous $\mathrm{AlCl}_{3}$ and then common de-ashing treatment (e.g., by washing with weak acid and deionized water) to attain a low AC of $\sim 790 \mathrm{ppm}$ was used as the starting material to prepare various synthetic pitches with tunable LC contents. About 400 $\mathrm{g}$ of NP was placed in a $1.5-\mathrm{L}$ stainless-steel autoclave reactor equipped with programmed heating, magnetic stirring, and pressure control. The reaction conditions of thermal polymerization were set at $400-440{ }^{\circ} \mathrm{C}$ for $4-10 \mathrm{~h}$ under a nitrogen pressure of 0-4 $\mathrm{MPa}$ similar to previous work (0 MPa refers to the simple closed-vessel experiment, disregarding a slight autogenous pressure owing to the volatile gases). ${ }^{29}$ The heating rate from room temperature to the reaction temperature was fixed at $3{ }^{\circ} \mathrm{C} / \mathrm{min}$ and the magnetic stirring speed was set at $750 \mathrm{rpm}$ for all experiments. Various SNPs with tunable LC percentages ranging from 0 to 98 vol \% could be readily obtained by adjusting the polycondensation conditions (i.e., the reaction temperature, time, and pressure). However, the synthetic pitches obtained with a low mesophase content of $\sim 30$ vol \% possessed a relatively low SP of $217{ }^{\circ} \mathrm{C}$. Especially, the SP of the synthetic pitches with a low mesophase content of $\sim 5$ vol \% (before the nitrogen-bubbling treatment) was measured to be as low as $\sim 140{ }^{\circ} \mathrm{C}$, which limited the high efficiency of oxidative stabilization. Thus, an air-blowing technique (i.e., oxidizing the NP at 350 and $380{ }^{\circ} \mathrm{C}$ for 6 and $5 \mathrm{~h}$, respectively, at a constant air flow of $1600 \mathrm{~mL} /$ min) under normal pressure in the same autoclave was adopted to speed up the cross-linking reaction and obtain the synthetic isotropic pitch (SIP) and low-mesophase-content pitch (e.g., 5 vol \%). When a melt-spinnable pitch with a low mesophase content was being studied, this enabled the requirement for high $\mathrm{SP}$ to be met, so as to allow faster oxidative stabilization.

A 100 vol \% LC pitch with a relatively low AC was purposely prepared in this work so as to have a complete comparison with the benchmark bulk mesophase pitch (sold under the trade name AR-MP, a commercial naphthalene-derived synthetic mesophase pitch produced by Mitsubishi Gas Chemical Corporation, its AC being only $30 \mathrm{ppm}$.). To achieve this purpose, the NP precursor was dissolved in a toluene solvent by further removing the insoluble matter (i.e., residual catalyst) via filtration treatment, and the resulting toluene-soluble (TS) fraction of NP with an AC as low as 170 ppm (as anticipated) was used to prepare this naphthalenebased mesophase pitch with a relatively high purity under a similar thermal polymerization condition.

4.2. Phase-Separation Control of the LC in SNPs and Their Melt-Spinnability. The prepared SNPs with various mesophase contents $(0-100 \mathrm{vol} \%)$ after the above-mentioned thermal polymerization were further pretreated in the autoclave with stirring and nitrogen bubbling at a flow rate of $500 \mathrm{~mL} / \mathrm{min}$ at $350{ }^{\circ} \mathrm{C}$ for $2-3 \mathrm{~h}$ to remove some low- 
molecular-weight components (i.e., volatile fractions) and concentrate the heavy fractions. This treatment procedure favored the improvement of pitch thermal stability and yielded melt-spinnable synthetic pitches with controllable SPs ranging from 210 to $290{ }^{\circ} \mathrm{C}$ and a representative range of mesophase contents (e.g., 5, 30, 50, 75, 98, and 100 vol \%). A static hotsettlement experiment in a vertical test tube $(\Phi \sim 2 \mathrm{~cm} \times 10$ $\mathrm{cm}$ ) for $6 \mathrm{~h}$ at a temperature $\sim 80{ }^{\circ} \mathrm{C}$ above the SP of the parent pitch (i.e., around the melt-spinning temperature) was conducted to study the phase-separation behavior of the LC in the SNPs.

The spinnable pitches with representative LC contents and suitable SPs were melt-extruded on a lab-scale mono-filament melt-spinning apparatus equipped with a round-shaped spinneret (the nozzle diameter was $0.2 \mathrm{~mm}$ and the length to diameter ratio of the nozzle was 2) at a spinning temperature of $\sim 80{ }^{\circ} \mathrm{C}$ above the corresponding SP of the parent pitch to form uniform pitch fibers with a large diameter of $\sim 28 \mu \mathrm{m} .{ }^{32}$ The estimation of the melt-spinnability of various SNPs is based primarily on the continuity of meltspinning (i.e., the duration without fiber break) and the homogeneity of the fiber diameter.

4.3. Preparation of Pitch-Based Carbon Fibers with Different Structures and Properties. The as-spun largediameter pitch fibers from the various SNPs were stabilized at an oxidation temperature of $\sim 20{ }^{\circ} \mathrm{C}$ below the corresponding SP of the parent pitch (i.e., if the SP of pitch precursor is 240 ${ }^{\circ} \mathrm{C}$, the selected oxidation temperature of pitch fibers is 220 ${ }^{\circ} \mathrm{C}$ ) for $\sim 24 \mathrm{~h}$ in flowing air with a flow rate of $500 \mathrm{~mL} / \mathrm{min}$, which is based on the premise of the use of the same pitch precursor and similar fiber diameter (despite the slight difference in the oxidative reactivity of various components in the spinnable pitches and small oxygen content in the oxidized pitch, e.g., SIP). The green pitch fibers were directly put into the pre-oxidation oven and then heated from room temperature to the oxidation temperature (e.g., $240{ }^{\circ} \mathrm{C}$ ) at a heating rate of $3{ }^{\circ} \mathrm{C} / \mathrm{min}$. After completion of the stabilization treatment, the stabilized pitch fibers were subsequently carbonized at $1000{ }^{\circ} \mathrm{C}$ for $1 \mathrm{~h}$ under a nitrogen atmosphere in a tube furnace using a heating rate of $1.0^{\circ} \mathrm{C} / \mathrm{min}^{33}$

4.4. Characterization of the SNPs and Their Derived Carbon Fibers. SNPs with various LC contents, and their derivative large-diameter carbon fibers, were observed under a Carl Zeiss AX10 polarized light microscope (PLM) in reflectance mode so as to image the optically anisotropic content of the LC (according to ASTM D4616-1995-R2018) and the optical texture of the SNPs and their derivative carbon fibers. ${ }^{34}$ The SPs of various pitch samples were measured on a programmable hot stage under a small amount of flowing nitrogen in the PLM; this had been compared and corrected to the molten flow distance-temperature curves of various pitches recorded by a CFT-100EX capillary rheometer under a flowing nitrogen atmosphere from room temperature to 380 ${ }^{\circ} \mathrm{C}$ at a constant heating rate of $5{ }^{\circ} \mathrm{C} / \mathrm{min}$. The solubility of the NP precursor and its thermal polymerization pitch products was analyzed by dividing the test sample into several components (i.e., cyclohexane soluble (CHS), cyclohexane insoluble and toluene soluble (CHI-TS), toluene insoluble (TI), toluene insoluble and quinoline soluble (TI-QS), and quinoline insoluble (QI)) by sequential extraction treatments. ${ }^{19}$ The solution-state ${ }^{1} \mathrm{H}$ nuclear magnetic resonance $\left({ }^{1} \mathrm{H} \mathrm{NMR}\right)$ spectra of various pitch samples were obtained using a Bruker DD2 $600 \mathrm{MHz}$ Advance NMR spectrometer.
The quantitative ${ }^{1} \mathrm{H}$ NMR spectra were recorded by dissolving samples in a D-substituted dimethyl sulfoxide (DMSO) solvent ( sample $/ \mathrm{DMSO}=100 \mathrm{mg} / 1 \mathrm{~mL}$ ) with tetramethylsilane as the standard chemical shift reference. The hydrogen distribution of the spectrum was calculated according to the definition of the Brown-Ladner method. ${ }^{35}$ The molecular weight and its distribution in the representative pitch samples were determined by a Bruker maXis ultra-high-resolution flight mass spectrometer (MS) using a nitrogen laser $(\lambda=337 \mathrm{~nm})$. The accelerating and detecting voltages were 20 and $1.6 \mathrm{kV}$, and the detection mode of positive ions was linear. Thermogravimetric (TG) and differential scanning calorimetry (DSC) analyses of various pitch samples were performed on an STA 449F3 TG-DSC analyzer under a flowing nitrogen atmosphere from room temperature to $1000{ }^{\circ} \mathrm{C}$ at a constant heating rate of $5{ }^{\circ} \mathrm{C} / \mathrm{min}$.

The morphology and microstructure of carbon fibers were observed with a TESCAN VEGA 3 scanning electron microscope (SEM). The tensile properties of the carbonized fibers were measured by single-filament testing according to ASTM standard D3822-07. The average value of 30 sets of parallel data with an error of less than $10 \%$ was adopted. ${ }^{32}$ The axial electrical resistivities of the carbon fibers were obtained by measuring the average value of 15-20 filaments with a BS407 precision millimicro ohmmeter using a standard fourprobe method at room temperature. The microcrystalline structure of carbon fiber samples in a powder form was determined by X-ray diffraction (XRD) analysis on a Philips $X^{\prime}$ PERT MPD PRO diffractometer. The microcrystalline parameters of various carbon fibers were calculated through a line-broadening analysis using the Scherrer equation. ${ }^{36}$

\section{ASSOCIATED CONTENT}

\section{SI Supporting Information}

The Supporting Information is available free of charge at https://pubs.acs.org/doi/10.1021/acsomega.0c03226.

Calculated hydrogen distribution of various pitches; optical texture of the SNPs after standing treatment; molten flow distance-temperature curves of various pitches; melt-spinnability of the thermally polymerized pitches; optical texture of the cross sections of carbon fibers derived from various pitches; XRD patterns of carbon fibers derived from various pitches; and microcrystalline parameters of carbon fibers made from various pitches (PDF)

\section{AUTHOR INFORMATION}

\section{Corresponding Authors}

Guanming Yuan - The State Key Laboratory of Refractories and Metallurgy and Hubei Province Key Laboratory of Coal Conversion \& New Carbon Materials, Wuhan University of Science and Technology, Wuhan 430081, China; (1) orcid.org/ 0000-0002-4693-8358; Email: yuanguanming@wust.edu.cn

Xuanke Li - The State Key Laboratory of Refractories and Metallurgy and Hubei Province Key Laboratory of Coal Conversion \& New Carbon Materials, Wuhan University of Science and Technology, Wuhan 430081, China; Email: xkli@ $21 \mathrm{cn} . \mathrm{com}$ 


\section{Authors}

Zheng Xue - Hubei Province Key Laboratory of Coal Conversion \& New Carbon Materials, Wuhan University of Science and Technology, Wuhan 430081, China

Zhengwei Cui - The State Key Laboratory of Refractories and Metallurgy and Hubei Province Key Laboratory of Coal Conversion \& New Carbon Materials, Wuhan University of Science and Technology, Wuhan 430081, China

Aidan Westwood - School of Chemical and Process Engineering, University of Leeds, Leeds LS2 9JT, United Kingdom

Zhijun Dong - The State Key Laboratory of Refractories and Metallurgy and Hubei Province Key Laboratory of Coal Conversion \& New Carbon Materials, Wuhan University of Science and Technology, Wuhan 430081, China

Ye Cong - The State Key Laboratory of Refractories and Metallurgy and Hubei Province Key Laboratory of Coal Conversion \& New Carbon Materials, Wuhan University of Science and Technology, Wuhan 430081, China

Jiang Zhang - The State Key Laboratory of Refractories and Metallurgy and Hubei Province Key Laboratory of Coal Conversion \& New Carbon Materials, Wuhan University of Science and Technology, Wuhan 430081, China

Hui Zhu - The State Key Laboratory of Refractories and Metallurgy and Hubei Province Key Laboratory of Coal Conversion \& New Carbon Materials, Wuhan University of Science and Technology, Wuhan 430081, China

Complete contact information is available at: https://pubs.acs.org/10.1021/acsomega.0c03226

\section{Author Contributions}

This manuscript was written through contributions of all authors. All authors have given approval to the final version of this manuscript.

\section{Notes}

The authors declare no competing financial interest.

\section{ACKNOWLEDGMENTS}

This work was sponsored by the National Natural Science Foundation of China (grant Nos. U1960106 and 51372177), the Key Laboratory of Hubei Province for Coal Conversion and New Carbon Materials (grant No. WKDM201701), the WUST National Defence Pre-research Foundation GF201808, and the China Scholarship Council Fund (No. 201808420114).

\section{REFERENCES}

(1) Brooks, J. D.; Taylor, G. H. The formation of graphitizing carbons from the liquid phase. Carbon 1965, 3, 185-193.

(2) Mochida, I.; Korai, Y.; Ku, C. H.; Watanabe, F.; Sakai, Y. Chemistry of synthesis, structure, preparation and application of aromatic-derived mesophase pitch. Carbon 2000, 38, 305-328.

(3) Hurt, R. H.; Chen, Z. Y. Liquid crystals and carbon materials. Phys. Today 2000, 53, 39-44.

(4) Singer, L. S. Carbon fibres from mesophase pitch. Fuel 1981, 60, 839-847.

(5) Matsumoto, T. Mesophase pitch and its carbon fibers. Pure Appl. Chem. 1985, 57, 1553-1562.

(6) Minus, M. L.; Kumar, S. The processing, properties, and structure of carbon fibers. JOM 2005, 57, 52-58.

(7) Frank, E.; Ingildeev, D.; Steudle, L. M.; Spörl, J. M.; Buchmeiser, M. R. Carbon fibers: precursor systems, processing, structure and properties. Angew. Chem., Int. Ed. 2014, 53, 5262-5298.
(8) Emmerich, F. G. Young's modulus, thermal conductivity, electrical resistivity and coefficient of thermal expansion of mesophase pitch-based carbons fibers. Carbon 2014, 68, 274-293.

(9) Bhatia, G.; Fitzer, E.; Kompalik, D. Mesophase formation in defined mixtures of coal tar pitch fractions. Carbon 1986, 24, 489494.

(10) Li, M.; Zhang, Y. D.; Yu, S. T.; Ding, J. W.; Bian, B.; Liu, D. Effects of in-process hydrogenation on mesophase development during the thermal condensation of petroleum aromatic-rich fraction. Energy Fuels 2018, 32, 5659-5663.

(11) Lamie, W. C.; Bruce, D. A.; Hoffman, W. P.; Thies, M. C. Kinetics of the catalytic polymerization of pyrene with $\mathrm{AlCl}_{3}$. Carbon 2018, 134, 1-8.

(12) Castro, L. D. D. Anisotropy and mesophase formation towards carbon fibre production from coal tar and petroleum pitches- a review. J. Braz. Chem. Soc. 2006, 17, 1096-1108.

(13) Li, M.; Zhang, Y. D.; Yu, S. T.; Xie, C. X.; Liu, D.; Liu, S. W.; Zhao, R. Y.; Bian, B. Preparation and characterization of petroleum based mesophase pitch by thermal condensation with in-process hydrogenation. RSC Adv. 2018, 8, 30230-30238.

(14) Bermudez, V.; Lukubira, S.; Ogale, A. A. Pitch Precursor-Based Carbon Fibers. In Comprehensive Composite Materials II; Zweben, C. H.; Beaumont, P. W. R., Eds.; Elsevier Science Ltd: Amsterdam, 2018; pp 41-65.

(15) Mochida, I.; Yoon, S. H.; Korai, Y. Mesoscopic structure and properties of liquid crystalline mesophase pitch and its transformation into carbon fiber. Chem. Rec. 2002, 2, 81-101.

(16) Mochida, I.; Sone, Y.; Korai, Y. Preparation and properties of carbonaceous mesophase-II highly soluble mesophase from ethylene tar modified using aluminum chloride as a catalyst. Carbon 1985, 23, $175-178$.

(17) Kumar, S.; Srivastava, M. Catalyzing mesophase formation by transition metals. J. Anal. Appl. Pyrolysis 2015, 112, 192-200.

(18) Moriyama, R.; Hayashi, J. I.; Suzuki, K.; Hiroshima, T.; Chiba, T. Analysis and modeling of mesophase sphere generation, growth and coalescence upon heating of a coal tar pitch. Carbon 2002, 40, $53-64$.

(19) Yuan, G. M.; Jin, Z.; Zuo, X. H.; Xue, Z.; Yan, F.; Dong, Z. J.; Cong, Y.; Li, X. K. Effect of carbonaceous precursors on the structure of mesophase pitches and their derived cokes. Energy Fuels 2018, 32, 8329-8339.

(20) Chen, L.; Fan, X. H.; Jiang, Z.; Ouyang, T.; Fei, Y. Q. Observation of "in-contact" characteristics of Brooks-Taylor mesophase spheres obtained by high-temperature centrifugation. Carbon 2016, 103, 421-424.

(21) Toshima, H.; Mochida, I.; Korai, Y.; Hino, T. Modification of petroleum-derived mesophase pitch by blending naphthalene-derived partially isotropic pitches. Carbon 1992, 30, 773-779.

(22) Blanco, C.; Fleurot, O.; Menéndez, R.; Santamar1'a, R.; Bermejo, J.; Edie, D. Contribution of the isotropic phase to the rheology of partially anisotropic coal-tar pitches. Carbon 1999, 37, $1059-1064$

(23) Blanco, C.; Santamarı'a, R.; Bermejo, J.; Menéndez, R. Separation and characterization of the isotropic phase and co-existing mesophase in thermally treated coal-tar pitches. Carbon 2000, 38, $1169-1176$.

(24) Ramjee, S.; Rand, B.; Focke, W. W. Low shear rheol haviour of two-phase mesophase pitch. Carbon 2015, 82, 368-380.

(25) Singer, L. S.; Lewis, I. C.; Greinke, R. A. Characterization of the phases in centrifuged mesophase pitches. Mol. Crys. Liq. Cryst. 1986, 132, 65-79.

(26) Kim, C. J.; Ryum, S. K.; Rhee, B. S. Properties of coal tar pitchbased mesophase separated by high-temperature centrifugation. Carbon 1993, 31, 833-838.

(27) Korai, Y.; Inoue, M.; Mochida, I.; Maeda, T. Modification of precursor pitch for general performance carbon fibre by blending naphthalene-derived isotropic and mesophase pitches. J. Mater. Sci. 1994, 29, 2911-2915. 
(28) Korai, Y.; Ishida, S.; Watanabe, F.; Yoon, S. H.; Wang, Y. G.; Mochida, I.; Kato, I.; Nakamura, T.; Sakai, Y.; Komatsu, M. Preparation of carbon fiber from isotropic pitch containing mesophase spheres. Carbon 1997, 35, 1733-1737.

(29) Mora, E.; Blanco, C.; Santamarı'a, R.; Granda, M.; Menéndez, R. A novel method to obtain a petroleum-derived mesophase pitch suitable as carbon fibre precursor. Carbon 2003, 41, 445-452.

(30) Yao, Y. B.; Chen, J. M.; Liu, L.; Dong, Y. M.; Liu, A. H. Tailoring structures and properties of mesophase pitch-based carbon fibers based on isotropic/mesophase incompatible blends. J. Mater. Sci. 2012, 47, 5509-5516.

(31) Yuan, G. M.; Li, X. K.; Xiong, X. Q.; Dong, Z. J.; Westwood, A.; Li, B. L.; Ye, C.; Ma, G. Z. A comprehensive study on the oxidative stabilization of mesophase pitch-based tape-shaped thick fibers with oxygen. Carbon 2017, 115, 59-76.

(32) Yuan, G. M.; Li, B. L.; Li, X. K.; Dong, Z. J.; Hu, W. J.; Westwood, A.; Cong, Y.; Zhang, J. Effect of liquid crystalline texture of mesophase pitches on the structure and property of large diameter carbon fibers. ACS Omega 2019, 4, 1095-1102.

(33) Yuan, G. M.; Li, X. K.; Dong, Z. J.; Westwood, A.; Rand, B.; Cui, Z. W.; Cong, Y.; Zhang, J.; Li, Y. J.; Zhang, Z. W.; et al. The structure and properties of ribbon-shaped carbon fibers with high orientation. Carbon 2014, 68, 426-439.

(34) Vieira, F. R.; Dutra, C. H. M. D. C.; Castro, L. D. D. Determining the anisotropic content in a petroleum pitchcomparison of centrifugation and optical microscopy techniques. Fuel 2011, 90, 908-911.

(35) Brown, J. K.; Ladner, W. R. A Study of the hydrogen distribution in coal-like materials by high-resolution nuclear magnetic resonance spectroscopy I- the measurement and interpretation of the spectra. Fuel 1960, 39, 79-86.

(36) Iwashita, N.; Park, C. R.; Fujimoto, H.; Shiraishi, M.; Inagaki, M. Specification for a standard procedure of X-ray diffraction measurements on carbon materials. Carbon 2004, 42, 701-714.

(37) Yang, H. X.; Han, H. X.; Wang, J. T.; Qiao, W. M.; Ling, L. C. Controllable synthesis of highly graphitizable pitches from 1methylnaphthalene via closed-system dehydrobromination. Energy Fuels 2018, 32, 11055-11066.

(38) Guo, J. G.; Zhu, H.; Xu, H. T.; Westwood, A.; Li, X. K. Spinnable mesophase pitch prepared via co-carbonization of fluid catalytic cracking decant oil and synthetic naphthalene pitch. Energy Fuels 2020, 34, 2566-2573.

(39) Thies, M. C. Fractionation and Characterization of Carbonaceous Pitch Oligomers: Understanding the Building Blocks for Carbon Materials. In Polymer Precursor-Derived Carbon; Naskar, A. K.; Hoffman, W. P., Eds.; ACS Symposium Series; American Chemical Society: Washington, D.C, 2014; pp 85-136.

(40) Zhang, W.; Andersson, J. T.; Rädera, H. J.; Müllena, K. Molecular characterization of large polycyclic aromatic hydrocarbons in solid petroleum pitch and coal tar pitch by high resolution MALDI ToF MS and insights from ion mobility separation. Carbon 2015, 95, $672-680$.

(41) Blanco, C.; Appleyard, S. P.; Rand, B. Study of carbon fibres and carbon-carbon composites by scanning thermal microscopy. $J$. Microsc. 2002, 205, 21-32.

(42) Takahashi, H.; Kuroda, H.; Akamatu, H. Correlation between stacking order and crystallite dimensions in carbons. Carbon 1965, 2, 432-433.

(43) Lavin, J. G.; Boyington, D. R.; Lahijani, J.; Nystem, B.; Issi, J. P. The correlation of thermal conductivity with electrical resistivity in mesophase pitch-based carbon fiber. Carbon 1993, 31, 1001-1002.

(44) Shimanoe, H.; Mashio, T.; Nakabayashi, K.; Inoue, T.; Hamaguchi, M.; Miyawaki, J.; Mochida, I.; Yoon, S. H. Manufacturing spinnable mesophase pitch using direct coal extracted fraction and its derived mesophase pitch based carbon fiber. Carbon 2020, 158, 922929. 